\title{
Alimentos fermentados y probióticos en niños. La importancia de conocer sus diferencias microbiológicas
}

\author{
Fermented foods and probiotics for children. The importance of \\ knowing their microbiological differences
}

a. Instituto de Lactología Industrial (INLAIN, CONICET-UNL),

Facultad de Ingeniería Química, Universidad

Nacional del Litoral.

b. Departamento

Materno-Infantil del Hospital Militar Central de Buenos Aires.

Correspondencia: Dr. Gabriel Vinderola: gvinde@fiq.unl.edu.ar

Financiamiento:

Proyecto PICT-2016-0256 (ANPCyT). "Desarrollo de biocultivos a partir de cepas autóctonas: hacia la autonomía en la producción tecnológica de microorganismos de interés en alimentos para humanos y animales", 2017-2020.

Conflicto de intereses: GV es investigador principal del Consejo Nacional de Investigaciones Científicas y Técnicas (CONICET) y profesor asociado de la Cátedra de Microbiología (Facultad de Ingeniería Química de la Universidad Nacional del Litoral, FIQ-UNL). GPM es investigador principal de múltiples estudios clínicos patrocinados por la industria farmacéutica. Ambos autores han realizado tareas de vinculación tecnológica (servicios tecnológicos, desarrollo de proyectos, consultorías y asesoramientos) con industrias de alimentos.

Estas actividades son intelectualmente independientes de la producción de manuscritos científicos y no influenciaron en el contenido de este trabajo.

\author{
Dr. Gabriel Vinderola y Dr. Gonzalo Pérez-Marc ${ }^{b}$
}

\section{RESUMEN}

La leche materna provee microorganismos que colonizan el intestino y programan el sistema inmunológico para desarrollar tolerancia oral. Entre los 6 meses de lactancia materna exclusiva y los 2 años de lactancia prolongada recomendada, la alimentación complementaria conlleva una reducción progresiva en el ingreso de microorganismos vivos al ecosistema intestinal. Esto se debe a que los alimentos en general -a diferencia de la leche materna- o se encuentran desprovistos de microorganismos $\mathrm{o}$, si los poseen, suelen inactivarse durante la cocción. Los alimentos fermentados y los probióticos podrían constituir una estrategia nutricional valiosa, dado que garantizarían la provisión de microorganismos vivos ante la reducción o interrupción anticipada de la lactancia. Los términos "alimentos fermentados" y "probióticos" no son sinónimos. La identidad microbiológica, la inocuidad y la existencia de estudios clínicos de eficacia para unos y otros son claves para entender sus diferencias y decidir una eventual recomendación alimentaria.

Palabras clave: microbiota, inmunidad, yogur, probióticos, alimentos fermentados.

http: / / dx.doi.org/10.5546/ aap.2021.56

Texto completo en inglés:

http:/ / dx.doi.org/10.5546/ aap.2021.eng.56

Cómo citar: Vinderola C, Pérez-Marc G. Alimentos fermentados y probióticos en niños. La importancia de conocer sus diferencias microbiológicas Arch Argent Pediatr 2021;119(1):56-61.

\section{Microbiota intestinal en los primeros 1000 días}

Los primeros 1000 días de vida de un/a niño/a constituyen el período que transcurre entre la fecundación y los 2 años de edad. A lo largo de este período (e incluso antes), y durante toda la vida, la fisiología humana se encuentra influenciada de diversas maneras por microorganismos. Es posible, incluso, que aquellos espermatozoides responsables de nuestro origen hayan interactuado con bacterias ya durante su viaje a través del canal vaginal, y que la fertilidad haya sido condicionada por la microbiota allí presente. ${ }^{1}$

Con el término "microbiota", se hace referencia al conjunto de microorganismos que colonizan nuestro cuerpo. La microbiota está compuesta, principalmente, por bacterias, aunque incluye también una gran cantidad de virus, hongos, levaduras, protozoos y arqueas. La microbiota intestinal es la más estudiada y la que concentra mayor diversidad y abundancia de especies bacterianas. El término "microbioma" hace referencia a los genes contenidos por los microorganismos de la microbiota. Si bien el término "microbiota" incluye el de "microbioma", solo el primero de ellos será el de preferencia en este artículo.

La microbiota es un "órgano difuso" que se hereda en forma conjunta de la madre, del entorno familiar y del medioambiente, que se establece y madura a nivel microbiológico durante 
los 2-3 primeros años de vida. En el contexto de embarazos saludables y normales, ${ }^{2}$ hay reportada presencia de material microbiano (células no viables, paredes celulares y ácido desoxirribonucleico-ADN-) en la placenta, el líquido amniótico, las membranas fetales y el tracto gastrointestinal fetal. Pero el principal aporte microbiano materno para la colonización intestinal del bebé tiene lugar con su paso a través del canal vaginal y por el aporte de leche materna. Otros factores que contribuyen a completar la colonización intestinal del bebé son la interacción con la familia y el medioambiente, así como el tipo de alimentación. ${ }^{3}$

Gracias a su composición rica en nutrientes, microorganismos y oligosacáridos (antes conocidos como "factores bifidogénicos"), la leche materna es el mejor alimento posible para el desarrollo de la microbiota intestinal y la maduración inmunológica del intestino. ${ }^{4}$ La rápida instalación de una microbiota estrictamente anaeróbica tiene importantes efectos sobre la salud intestinal, debido a la producción de ácidos grasos de cadena corta; el ácido butírico es un importante agente antiinflamatorio. ${ }^{5}$ El parto prematuro, por ejemplo, retrasa significativamente la dominancia por anaerobios estrictos. ${ }^{6}$ El nacimiento por cesárea y el uso de antibióticos se asocian a una mayor prevalencia, a lo largo de la infancia, de asma, artritis juvenil, enfermedades inflamatorias intestinales, deficiencias inmunológicas, sobrepeso, obesidad, alergias, eczemas e infecciones entéricas y respiratorias. ${ }^{7}$

Los mecanismos que explicarían estos desórdenes inflamatorios e inmunológicos se encuentran relacionados con la colonización aberrante del intestino, en la que (en lugar de los habituales y beneficiosos Lactobacillus, Bifidobacterium o Faecalibacterium prausnitzii), prevalecerían microorganismos proinflamatorios, como Enterococcus faecalis, Clostridium difficile, Campylobacter, Methanobrevibacter smithii. ${ }^{3}$ Bifidobacterium es el género dominante en el intestino del bebé sano durante el primer año de vida. Su dinámica de colonización intestinal se ve afectada en niños nacidos por cesárea, pues se enlentece durante los primeros meses posnacimiento. ${ }^{8}$ Este enlentecimiento es crucial en el desarrollo del sistema inmunológico, por lo que el nacimiento por cesárea tendría un impacto relevante en la predisposición a desarrollar patologías inmunológicas. ${ }^{9}$ Una publicación reciente en los Archivos Argentinos de Pediatría resalta la importancia de fomentar la lactancia materna; de promover el uso racional de antibióticos en el período perinatal y de disminuir el número de nacimientos por cesárea, dadas sus claras repercusiones sobre la microbiota del recién nacido. ${ }^{10}$

\section{El concepto nutricional de una dosis diaria de microorganismos}

En 1907, el inmunólogo ganador del Premio Nobel Ilya Metchnikoff publicó su tratado sobre la longevidad, titulado La prolongación de la vida: Estudios optimistas. Propuso, sobre la base de una serie de observaciones, que los campesinos búlgaros, que solían ingerir una gran cantidad de productos lácteos fermentados, tenían vidas largas y saludables. Atribuyó esto a los beneficios de las bacterias contenidas en el yogur. ${ }^{11}$ De estas ideas proviene el nombre de la especie Lactobacillus bulgaricus, una de las dos bacterias del yogur.

La ingesta habitual de microorganismos puede proporcionar múltiples beneficios, como el mantenimiento de la salud y la prevención de diversas patologías. ${ }^{12}$ La leche materna, cargada de hasta $10^{7}$ microorganismos por mililitro, ${ }^{13}$ es el primer alimento rico en microorganismos al que se está expuesto. Por cuestiones culturales, se ha incorporado con firmeza la idea de que la ingesta de un número relativamente pequeño de microorganismos patógenos, en ocasiones, presentes en alimentos es capaz de provocar infecciones de diversa gravedad, pero, en cierto modo, hay aún escepticismo respecto de la idea contraria: que existen microorganismos que, consumidos en cantidades adecuadas, pueden ejercer efectos muy beneficiosos para la salud.

Nuestra alimentación ancestral ha sido rica en microorganismos. Sin embargo, la urbanización de nuestros hábitos alimentarios ha reducido en forma significativa la riqueza microbiana a la se está expuesto a diario. ${ }^{14}$ Esta merma en la exposición a microorganismos se ha asociado a la proliferación de enfermedades crónicas. ${ }^{15}$ ¿Cómo lograr, entonces, que toda una generación educada en la importancia de la higiene y la limpieza, y para la que el único microorganismo bueno es aquel que está muerto, pueda comprender la relevancia que tiene la presencia de estos en la dieta habitual? Una manera podría ser a partir de la adaptación del concepto de valor diario de referencia (VDR), que es la cantidad promedio de un determinado nutriente que una persona sana debe ingerir 
diariamente para mantener un correcto estado de salud. En este contexto, se podría hablar de un "VDR de microorganismos", de la misma manera en la que se habla de macronutrientes, vitaminas y oligoelementos (https: / / isappscience.org/ rda-for-microbes/). Así, cabría preguntarse: ¿Qué alimentos podrían ofrecer un VDR de microorganismos seguros en la alimentación complementaria de un/a niño/a?

\section{Alimentos fermentados y probióticos}

Existe, fundamentalmente, en las redes sociales, un uso indistinto de los términos "probiótico" y "alimento fermentado". Es incorrecto y puede generar dudas o confusión entre profesionales de la salud (nutricionistas, gastroenterólogos, pediatras, geriatras, etc.) al momento de realizar una recomendación alimentaria acerca de estos productos. No todos los alimentos fermentados son probióticos ni todos los probióticos se encuentran disponibles exclusivamente en los alimentos fermentados.

Los alimentos fermentados son aquellos obtenidos mediante el desarrollo controlado de microorganismos (bacterias, levaduras, hongos) y su acción enzimática sobre un sustrato alimenticio, que lo transforma en un producto diferente al material de partida. La fermentación modifica las propiedades reológicas (viscosidad) y sensoriales del producto (aroma, sabor), mejora la digestibilidad y la biodisponibilidad de algunos nutrientes, extiende la vida útil (debido a la producción de ácido láctico) y puede conferirle al alimento ciertas propiedades benéficas para la salud. ${ }^{16,17}$ Los alimentos fermentados pueden provenir de la fermentación de la leche (yogur, kéfir), de vegetales (chucrut, kimchi), de cereales (cerveza, avena fermentada) y de legumbres o frutas (bebidas alcohólicas). El perfil nutricional de los alimentos fermentados es muy diverso, y un análisis exhaustivo está fuera del objetivo de este trabajo. Desde un punto de vista microbiológico, la fermentación puede llevarse a cabo con los microorganismos naturalmente presentes en los sustratos por fermentar (chucrut, kimchi), a través de consorcios microbianos presentes en gránulos o "madres" que se agregan al sustrato por fermentar (kéfir, kombucha) o mediante el uso de cultivos microbianos seleccionados (yogur, quesos).

Por su parte, los probióticos fueron definidos por la Organización Mundial de la Salud en 2002 de la siguiente manera: ‘microorganismos vivos que, cuando son administrados en cantidades adecuadas, ejercen un efecto benéfico sobre la salud'. Esta definición fue ratificada por un grupo de expertos de la Asociación Científica Internacional de Probióticos y Prebióticos (International Scientific Association for Probiotics and Prebiotics, ISAPP) ${ }^{18} \mathrm{y}$ fue incorporada en 2011 al Código Alimentario Argentino. La definición de "probiótico" implica tres aspectos: 1) que se trate de un microorganismo -o mezcla de microorganismos-definido microbiológicamente, es decir, del que se conozcan el género, la especie y la cepa; 2) que se trate de microorganismos que se encuentren vivos al momento de ser consumidos; y 3) que exista, al menos, un estudio clínico de eficacia que demuestre sus efectos beneficiosos. ${ }^{19}$ Los probióticos pueden estar presentes en la forma de suplementos alimenticios (cápsulas, pastillas o suspensiones acuosas) o incorporados en alimentos, como en algunas fórmulas infantiles, yogures, quesos frescos o jugos de fruta. ${ }^{20}$

\section{Yogur y yogur con probióticos}

El yogur es el producto que se obtiene de la fermentación de la leche con dos bacterias específicas, llamadas Streptococcus thermophilus y Lactobacillus delbrueckii, subsp. bulgaricus. Estas se encargan de fermentar parcialmente la lactosa para acidificar la leche. Los yogures, en general, son mejor tolerados que la leche por los sujetos mal absorbedores o intolerantes a la lactosa, debido a que parte de la lactosa se consume durante la fermentación. A su vez, una hidrólisis adicional de la lactosa tiene lugar una vez ingerido el yogur; la lactosa se sigue hidrolizando con la enzima lactasa liberada por las bacterias lácticas al ser lisadas por los jugos biliares.

De todos modos, para aquellas personas con un alto grado de intolerancia a la lactosa, existen yogures industriales sin lactosa. Incluso es posible elaborar yogur casero con leche deslactosada, ya que las bacterias lácticas son capaces de hallar, en la glucosa y la galactosa (azúcares provenientes de la hidrólisis de la lactosa en las leches deslactosadas industriales), los azúcares necesarios para llevar a cabo la fermentación. Es oportuno mencionar también que, debido a los procesos de pasteurización a los que es sometida la leche antes de la fermentación, el yogur no es un potencial vehículo de la bacteria causante del síndrome urémico hemolítico. ${ }^{21}$

En algunos casos, la composición microbiológica de los yogures puede enriquecerse 
con el agregado de bacterias probióticas, que son cepas específicas de los géneros Lactobacillus y Bifidobacterium; L. casei, L. paracasei, L. rhamnosus o B. lactis son las especies más utilizadas. Las especies microbianas utilizadas para elaborar yogur (o utilizadas como probióticos) poseen estatus de seguridad Generally Recognized as Safe (GRAS) según la Food and Drug Administration (FDA) de Estados Unidos o Qualified Presumption of Safety (QPS), presunción calificada de seguridad, de la Agencia Europea de Seguridad Alimentaria (European Food Safety Authority, EFSA).

Un ejemplo de yogur con probióticos es el desarrollado por investigadores del Centro de Referencia para Lactobacilos (CERELA), Tucumán, y diferentes instituciones de Gobierno, para ser utilizado en un programa social originado en la provincia de Tucumán y que lleva más de 10 años de aplicación en escuelas de varias provincias del Noroeste Argentino (NOA) y municipios bonaerenses. ${ }^{22}$ Este caso es un ejemplo de coincidencia entre los términos "alimento fermentado" y "probiótico", ya que se trata de un producto que es el resultado de la fermentación de la leche con Streptococcus thermophilus y Lactobacillus delbrueckii subsp. bulgaricus, al que luego se le agrega el probiótico Lactobacillus rhamnosus CRL1505, que posee -como es indicado- su correspondiente estudio de eficacia en niños para la prevención de infecciones del árbol respiratorio, entre otros efectos.

Este es un ejemplo de esos casos en los que se cumplen adecuadamente las condiciones de la definición de probiótico: existe una cepa definida, es posible verificar su viabilidad mediante el uso de medios de cultivo específicos ${ }^{23,24}$ y hay un estudio clínico de eficacia que respalda su indicación. ${ }^{25} \mathrm{Al}$ estar incluido en un yogur, es posible denominar este producto como "alimento fermentado probiótico". Otro tipo de alimento fermentado probiótico que no es de base láctea es un producto disponible en los países nórdicos y que consiste en avena fermentada con la cepa probiótica Lactobacillus plantarum $299 \mathrm{v},{ }^{26}$ la cual se combina luego con diferentes pulpas de frutas para dar un producto tipo "batido" o smoothie. ${ }^{27}$

Un estudio demostró que la incorporación de yogures a la dieta proveía más de 1000 veces microorganismos que una alimentación que no incorporaba alimentos fermentados. ${ }^{28}$ Los probióticos también pueden estar incluidos en alimentos no fermentados, ${ }^{29}$ como en ciertas fórmulas infantiles ${ }^{30} \mathrm{o}$ en suplementos dietarios. ${ }^{31}$
En estos casos, se utilizan, además de Lactobacillus y Bifidobacterium, otras especies, como Lactobacillus reuteri, Saccharomyces boulardii o Bacillus coagulans. En relación con la terapéutica pediátrica, se han desarrollado probióticos que demostraron diferentes niveles de eficacia para la prevención de la diarrea asociada a antibióticos ${ }^{32}$ y de los cólicos infantiles. ${ }^{33}$

Sociedades científicas internacionales han emitido documentos guías para el uso de probióticos, como la Organización Mundial de Gastroenterología (https: / / www . worldgastroenterology.org/guidelines/globalguidelines / probiotics-and-prebiotics / probioticsand-prebiotics-english), la Sociedad Europea para la Nutrición y Gastroenterología Pediátrica, y, más recientemente, la Asociación Americana de Gastroenterología. ${ }^{34}$

\section{Alimentos fermentados con microbiota indefinida}

Los alimentos fermentados, como el kéfir de leche, el kéfir de agua, la kombucha, el chucrut o el kimchi, son alimentos indefinidos desde el punto de vista microbiológico, ya que se elaboran a partir de la fermentación espontánea del material de partida (con las bacterias y levaduras naturalmente presentes en el sustrato) o, como es el caso del kéfir y la kombucha, a partir de una comunidad microbiana compleja asociada a los gránulos de kéfir ${ }^{35} \mathrm{o}$ a la "madre" (mucílago) de la kombucha. ${ }^{17}$ Estos alimentos fermentados están integrados por un número variable de especies y cepas de bacterias lácticas, bacterias acéticas y levaduras, según el caso. Los gránulos de kéfir provenientes de diferentes orígenes tienen composición microbiológica diferente, ${ }^{36}$ por lo que producen alimentos con distinta composición microbiológica.

Además, a lo largo de los sucesivos repiques (o subcultivos de los gránulos), la comunidad microbiana va modificando progresivamente su composición. ${ }^{37}$ En este contexto, no es posible conocer a priori la identidad de los microorganismos presentes en un kéfir elaborado de forma casera o artesanal. Adicionalmente, es importante tener en cuenta que tanto el kéfir de leche como el kéfir de agua, debido a la presencia de levaduras, pueden contener del $0,5 \%$ al $3 \%$ de alcohol, ${ }^{35,38,39}$ por lo que su consumo estaría desaconsejado durante el embarazo, la lactancia y durante la infancia. La identidad de los microorganismos que integran un producto que se denomine "probiótico" es uno de los requisitos 
necesarios para conocer de qué microorganismo se trata y, en función de esto, poder determinar qué estudios respaldan su capacidad probiótica. Sin embargo, el hecho de que alimentos fermentados con composición indefinida (como el kéfir, el chucrut o la kombucha) no puedan denominarse técnicamente "probióticos" no implica que no tengan potenciales efectos beneficiosos para la salud. Estos podrían, a lo sumo, inferirse a partir de estudios realizados con alimentos similares, ${ }^{35}$ pero no evitaría que subsistieran las dudas acerca de la posibilidad de asignarles las mismas conclusiones acerca de su seguridad e inocuidad.

\section{CONCLUSIÓN}

La leche materna es el mejor alimento para el bebé, tanto por su composición nutricional como por el aporte de microorganismos que conformarán su microbiota. Estos cumplen un papel fundamental en la modulación de la respuesta inmune de la mucosa intestinal para el desarrollo de la tolerancia oral y en la prevención de enfermedades crónicas. La alimentación complementaria supone una disminución del flujo de microorganismos vivos para el intestino, especialmente, si se discontinúa la lactancia en forma temprana. El uso excesivo de antibióticos durante la primera infancia, así como el creciente número de nacimientos por cesárea, han redundado en una alteración de la abundancia y diversidad de la microbiota de los lactantes. Debido a esto, el ingreso de microorganismos con la alimentación podría ocupar, al menos, en forma temporaria, los nichos ecológicos intestinales y ejercer algunas de las funciones naturales de los miembros indígenas de la microbiota.

Tanto los probióticos como ciertos alimentos fermentados serían fuente de microorganismos seguros y de identidad conocida, con amplios beneficios locales (a nivel intestinal) y sistémicos. Los términos "probiótico" y "alimento fermentado" no siempre son intercambiables. Ciertos yogures y fórmulas infantiles poseen probióticos. En estos casos, es posible conocer la identidad microbiológica de estos productos. Tanto los microorganismos del yogur como los cultivos con estudios clínicos que demuestran su carácter probiótico presentes en yogures o fórmulas infantiles tienen estatus GRAS o QPF de seguridad. La identidad e inocuidad microbiológica es un factor importante para tener en cuenta por los profesionales de la salud al momento de hacer recomendaciones alimentarias para incorporar microorganismos vivos a la alimentación complementaria durante la primera infancia.

\section{REFERENCIAS}

1. García-Velasco JA, Menabrito M, Catalán IB. What fertility specialists should know about the vaginal microbiome: a review. Reprod Biomed Online. 2017; 35(1):103-12.

2. Kuperman AA, Koren O. Antibiotic use during pregnancy: How bad is it? BMC Med. 2016; 14(1):91.

3. Milani C, Duranti S, Bottacini F, Casey E, et al. The First Microbial Colonizers of the Human Gut: Composition, Activities, and Health Implications of the Infant Gut Microbiota. Microbiol Mol Biol Rev. 2017; 81(4):e00036-17.

4. Doare KL, Holder B, Bassett A, Pannaraj PS. Mother's Milk: A purposeful contribution to the development of the infant microbiota and immunity. Front Immunol. 2018; 9:361.

5. Houghteling PD, Walker WA. Why is initial bacterial colonization of the intestine important to infants' and children's health? J Pediatr Gastroenterol Nutr. 2015; 60(3):394-307.

6. Arboleya S, Solís G, Fernández N, De los Reyes-Gavilán $\mathrm{CG}$, et al. Facultative to strict anaerobes ratio in the preterm infant microbiota: A target for intervention? Gut Microbes. 2012;3(6):583-8.

7. TamburiniS, Shen N, Wu HC, ClementeJC. The microbiome in early life: Implications for health outcomes. Nat Med. 2016; 22(7):713-22.

8. Reyman M, Van Houten MA, Van Baarle D, Bosch AA, et al. Impact of delivery mode-associated gut microbiota dynamics on health in the first year of life. Nat Commun. 2019; 10(1):4997.

9. Francino MP. Birth Mode-Related Differences in Gut Microbiota Colonization and Immune System Development. Ann Nutr Metab. 2018; 73(Suppl 3):12-6.

10. Uberos J. Microbiota perinatal: Revisión de su importancia en la salud del recién nacido. Arch Argent Pediatr. 2020; 118(3):e265-70.

11. Reid G. Probiotics: Definition, scope and mechanisms of action. Best Pract Res Clin Gastroenterol. 2016; 30(1):17-25.

12. Sanders ME, Benson A, Lebeer S, Merenstein DJ, et al. Shared mechanisms among probiotic taxa: implications for general probiotic claims. Curr Opin Biotechnol. 2018; 49:207-16.

13. Boix-Amorós A, ColladoMC, Mira A. Relationship between milk microbiota, bacterial load, macronutrients, and human cells during lactation. Front Microbiol. 2016; 7:492.

14. Logan AC, Katzman MA, Balanzá-Martínez V. Natural environments, ancestral diets, and microbial ecology: Is there a modern "paleo-deficit disorder"? Part II. J Physiol Anthropol. 2015; 34(1):9.

15. Kramer A, Bekeschus S, Bröker BM, Schleibinger H, et al. Maintaining health by balancing microbial exposure and prevention of infection: The hygiene hypothesis versus the hypothesis of early immune challenge. J Hosp Infect. 2013; 83(Suppl 1):S29-34.

16. DiCagno R, Coda R, De Angelis M, Gobbetti M. Exploitation of vegetables and fruits through lactic acid fermentation. Food Microbiol. 2013; 33(1):1-10.

17. Dimidi E, Cox SR, Rossi M, Whelan K. Fermented foods: Definitions and characteristics, impact on the gut microbiota and effects on gastrointestinal health and disease. Nutrients. 2019; 11(8):1806.

18. Hill C, Guarner F, Reid G, Gibson GR, et al. Expert consensus document: The international scientific association for probiotics and prebiotics consensus statement on the scope and appropriate use of the term probiotic. Nat Rev Gastroenterol Hepatol. 2014; 11(8):506-14. 
19. Reid G, Gadir AA, Dhir R. Probiotics: Reiterating what they are and what they are not. Front Microbiol. 2019; 10:424.

20. Fenster K, Freeburg B, Hollard C, Wong C, et al. The production and delivery of probiotics: A review of a practical approach. Microorganisms. 2019; 7(3):83.

21. Vinderola G, Rivas M. Síndrome Urémieo Hemolítico y yogur: entre la creencia popular y la evidencia científica. Rev Chil Nutr. 2020; 47(1):148-52.

22. Bortza G, Thomas H. Biotechnologies for inclusive development: Scaling up, knowledge intensity and empowerment (the case of the probiotic yoghurt 'yogurito' in argentina). Innov Dev. 2017; 7(1):37-61.

23. Vinderola CG, Reinheimer JA. Culture media for the enumeration of Bifidobacterium bifidum and Lactobacillus acidophilus in the presence of yoghurt bacteria. Int Dairy J. 1999; 9(8):497-505.

24. Vinderola G, Reinheimer J, Salminen S. The enumeration of probiotic issues: From unavailable standardised culture media to a recommended procedure? Int Dairy J. 2019; 96:58-95.

25. Villena JC, Salva MS, Nuñez MS, Corzo J, et al. Probiotics for Everyone! The Novel Immunobiotic Lactobacillus rhamnosus CRL1505 and the Beginning of Social Probiotic Programs in Argentina. Int J Biotechnol Wellness Ind. 2012; 1(3):189-98.

26. Molin G. Probiotics in foods not containing milk or milk constituents, with special reference to Lactobacillus plantarum 299v. Am J Clin Nutr. 2001; 73(2 Suppl):S380-5.

27. McNaught CE, Woodcock NP, Anderson ADG, MacFie J. A prospective randomised trial of probiotics in critically ill patients. Clin Nutr. 2005; 24(2):211-9.

28. Lang JM, Eisen JA, Zivkovic AM. The microbes we eat: Abundance and taxonomy of microbes consumed in a day's worth of meals for three diet types. Peer J. 2014; 2:e6590.
29. Hojsak I, Fabiano V, Pop TL, Goulet O, et al. Guidance on the use of probiotics in clinical practice in children with selected clinical conditions and in specific vulnerable groups. Acta Paediatr. 2018; 107(6):927-37.

30. Green Corkins K, Shurley T. What's in the Bottle? A Review of Infant Formulas. Nutr Clin Pract. 2016; 31(6):723-9.

31. Sreeja V, Prajapati JB. Probiotic Formulations: Application and Status as Pharmaceuticals-A Review. Probiotics Antimicrob Proteins. 2013; 5(2):81-91.

32. Szajewska H, Canani RB, Guarino A, Hojsak I, et al. Probiotics for the prevention of antibiotic-associated diarrhea in children. J Pediatr Gastroenterol Nutr. 2016; 62(3):495-506.

33. Urbańska M, Szajewska H. The efficacy of Lactobacillus reuteri DSM 17938 in infants and children: a review of the current evidence. Eur J Pediatr. 2014; 173(10):1327-37.

34. Su GL, Ko CW, Bercik P, Falck-Ytter Y, et al. AGA Clinical Practice Guidelines on the Role of Probiotics in the Management of Gastrointestinal Disorders. Gastroenterology. 2020; S0016-5085(20)34729-6.

35. Rosa DD, Dias MMS, Grześkowiak ŁM, Reis SA, et al. Milk kefir: Nutritional, microbiological and health benefits. Nutr Res Rev. 2017; 30(1):82-96.

36. Plessas S, Nouska C, Mantzourani I, Kourkoutas Y, et al. Microbiological exploration of different types of Kefir grains. Fermentation. 2017; 3(1).

37. Gao W, Zhang L, Feng Z, Liu H, et al. Microbial diversity and stability during primary cultivation and subcultivation processes of Tibetan kefir. Int J Food Sci Technol. 2015; 50(6):1468-76.

38. Laureys D, De Vuyst L. Microbial species diversity, community dynamics, and metabolite kinetics of water kefir fermentation. Appl Environ Microbiol.2014; 80(8):256472.

39. Laureys D, De Vuyst L. The water kefir grain inoculum determines the characteristics of the resulting water kefir fermentation process. J Appl Microbiol.2017; 122(3):719-732. 